\section{Leishmania infantum and Epstein-Barr virus co-infection in a patient with hemophago- cytosis}

\author{
Zied Gaifer, ${ }^{1}$ \\ Mohamed-Rachid Boulasse| $\left.\right|^{2,3}$ \\ 'Department of Medicine, ${ }^{2}$ Department of \\ Hematology, ${ }^{3}$ Department of Allied \\ Health Sciences, Sultan Qaboos \\ University, Sultanate of Oman
}

\section{Abstract}

The authors describe a rare case of a 27year old previously healthy male presenting with high grade fever, pancytopenia, hepatosplenomegaly, high levels of ferritin and triglyceride, suggesting a diagnosis of hemophagocytic lymphohistiocytosis (HLH) syndrome. Other investigations showed a positive Leishmania infantum serology and high Epstein-Barr virus (EBV) viremia. The diagnosis of a visceral leishmaniasis was confirmed by bone morrow biopsy, which showed Leishman-Donovan bodies and evidence of HLH. The patient received liposomal amphotericin B and he had a complete resolution of his symptoms and clearance of EBV viremia. This case of HLH associated with visceral leishmaniasis and EBV co-infection raises the question about the significance of $\mathrm{EBV}$ in patients with HLH. The treatment of actual etiological agent can lead to complete cure while using current recommend chemotherapy for HLH-related EBV in a patient with hidden infection may have deleterious effects.

\section{Introduction}

Association among Leishmania infantum, Epstein-Barr virus (EBV) co-infection and hemophagocytic lymphohistiocytosis (HLH) is rare in adults. Misdiagnosis of visceral leishmaniasis as a case of EBV-related HLH has serious consequences on patient's clinical outcome. Therefore, a thorough investigation for the underlying etiology for EBV-related HLH should be carefully carried out to avoid unnecessary treatment with chemotherapy. Herein the authors provide clinical evidence that liposomal amphotericin B can effectively treat visceral leishmaniasis leading to improvement in cellular responsiveness of the host to control EBV viral replication.

\section{Case Report}

A 27-year old previously healthy male was hospitalized for high grade fever that started three weeks prior to his admission. His fever was associated with chills and drenching sweat. During his illness he lost $10 \mathrm{~kg}$ of his weight, visited several medical clinics and received antibiotics without improvements in his symptoms. He used to smoke one pack of cigarette per day but denied any alcohol or drug use. He travelled to Yemen seven months prior to his admission to Sultan Qaboos University Hospital, Sultanate of Oman. The patient used to drink raw camel milk and his family history was noncontributory.

At time of admission to the hospital, physical examination showed a temperature of $40.4^{\circ} \mathrm{C}\left(104.7^{\circ} \mathrm{F}\right)$, heart rate 125 beat per minute and normal blood pressure. Neck exam showed multiple small non-tender lymph nodes in posterior cervical areas. Abdominal exam was significant for soft non-tender enlarged liver and spleen. The rest of his physical exam was unremarkable. At initial hospitalization blood studies showed pancytopenia with hemoglobin level of $6.6 \mathrm{~g} / \mathrm{dL}$, white blood count $1.5 \times 10^{9} / \mathrm{L}$ and platelet count of $69 \times 10^{9} / \mathrm{L}$. The blood film confirmed the pancytopenia, and further showed anisocytosis and basophilic stippling without any malaria parasites. Serum ferritin and triglycerides were high with values of $74,758 \mu \mathrm{g} / \mathrm{L}$ and 4.0 $\mathrm{mmol} / \mathrm{L}$, respectively. The liver function tests were mildly elevated with normal serum urea, electrolytes and coagulation profile. Blood, urine and respiratory cultures all were reported as no growth. Serologic tests for Brucella, Coxiella Burnettii, human immunodeficiency virus, viral hepatitis all were negative. Blood polymerase chain reaction for EBV showed a viral load of 13,559 copies $/ \mathrm{mL}$, while cytomegalovirus was undetectable. Bone morrow aspirate and biopsy both revealed haemophagocytosis and Leishman-Donovan bodies' consistence with a visceral leishmaniasis. Serum Leishmania infantum by enzymelinked immunosorbent assay was positive. The computed tomography of the chest and abdomen revealed a significant hepatosplenomegaly while transthoracic echocardiology was normal. At admission the patient was started on oral doxycycline $100 \mathrm{mg}$ twice daily as the diagnosis of $Q$ fever infection was suspected based on a history of animal exposure and an equivocal ELISA test for Q fever serology (Coxiella Burnettii serology test) that was done in other hospital. However, two days later in our hospital a repeated ELISA serology testing for $Q$ fever was negative and doxycycline was stopped. In the same day patient was put on gentamicin $370 \mathrm{mg}$ and rifampicin $600 \mathrm{mg}$ daily to cover the possibility of brucellosis
Correspondence: Zied Gaifer, Department of Medicine, Sultan Qaboos University Hospital, AlKhod, PO 123, Muscat, Sultanate of Oman.

E-mail: gaifer@squ.edu.om

Key words: Leishmania infantum, Epstein-Barr virus, coinfection, hemophagocytosis

Contributions: the authors contributed equally.

Conflict of interest: the authors declare no potential conflict of interest.

Received for publication: 16 April 2016. Revision received: 4 October 2016.

Accepted for publication: 16 October 2016.

This work is licensed under a Creative Commons Attribution-NonCommercial 4.0 International License (CC BY-NC 4.0).

(C) Copyright Z. Gaifer and M-R. Boulassel, 2016 Licensee PAGEPress, Italy

Infectious Disease Reports 2016; 8:6545

doi:10.4081/idr.2016.6545

since the patient used to drink raw camel milk. The patient received three days of gentamicin and rifampicin with no improvement in his symptoms and the diagnosis of brucellosis was excluded when Brucella blood culture and serology test both reported negative. At day five of admission the patient found have EBV viral load of 13,559 copies/mL. Due to high EBV viral load and pancytopenia the patient underwent bone marrow aspiration and biopsy to exclude EBV-related lymphoproliferative disorders but interestingly bone marrow aspirate showed Leishman-Donovan bodies suggesting a diagnosis of visceral leishmaniasis which was then confirmed by bone marrow biopsy and Leishmania serology. Immediately the patient was started on liposomal amphotericin B with the dose of $3 \mathrm{mg} / \mathrm{kg}$ once daily for five consecutive days as treatment for visceral leishmaniasis. Additional doses were also given at days 10,17, 24, 31, and 38 of starting liposomal amphotericin therapy. After receiving five doses of liposomal amphotericin B the patient became afebrile and all his symptoms improved but he remained neutropenic. Fever reoccurred again at day 8 of liposomal amphotericin therapy, patient was put empirically on piperacillin-tazobactam. Repeated blood, urine and sputum cultures were negative. Piperacillin-tazobactam was stopped at day 18 of starting liposomal amphotericin B when he became afebrile. Platelet count returned to normal after one week, while hemoglobin and white blood cells normalized after three weeks of liposomal amphotericin therapy.

Patient was discharged in a good clinical condition to continue three more doses of lipo- 
somal amphotericin B as outpatient. After completion of therapy patient was seen in infectious diseases clinic for regular followups at 1,3 and 6 months after hospital discharge. He had complete resolution of his previous symptoms. Six months after admission repeated EBV viral load was undetectable (Figure 1) and serum triglycerides and ferritin both returned to normal levels.

\section{Discussion}

HLH is rare lethal disease that occurs due to uncontrolled proliferation of T-lymphocytes and macrophages leading to phagocytosis of blood cells and infiltration of the reticuloendothelial system. ${ }^{1}$ Etiologically, HLH is classified as either primary or secondary. Primary HLH usually occurs in children and it is genetically mediated. Secondary HLH is associated with infections, malignancies or auto-immune diseases $^{2}$

Diagnosis of HLH often made by meeting one of the following criteria: a molecular diagnosis consistent with HLH syndrome or having five out of eight of the following: fever, splenomegaly, bicytopenia, high ferritin, high triglyceride or low fibrinogen, low/absent NKcell-activity, high-soluble interleukin-2-receptor levels, and histopathological evidence of hemophagocytosis. ${ }^{3}$

This report describes a patient who developed visceral leishmaniasis associated with

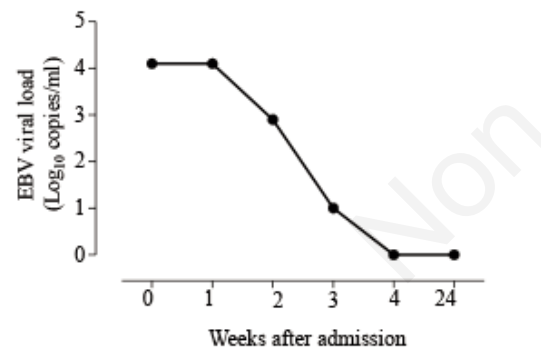

Figure 1. Changes of Epstein-Barr virus viral load following liposomal amphotericin B therapy.
EBV co-infection and HLH. To our knowledge this is the first case report showing that treatment with liposomal amphotericin B can effectively treat visceral leishmaniasis leading to improvement in cellular responsiveness of the host to control EBV viremia and dramatic resolution of clinical and laboratory abnormalities of HLH in an adult patient. Two cases of leishmaniasis with EBV co-infection were reported in children where a complete resolution of HLH syndrome was noticed with amphotericin B. Koliou et al reported on a 9-month-old girl with HLH associated with EBV and leishmaniasis coinfection, and who was successfully treated with liposomal amphotericin B. ${ }^{4}$ Similarly, Domínguez-Pinilla et al. reported on a 22-month-old girl who was diagnosed with leishmania and EBV coinfection and was given 7-day course of liposomal amphotericin B. Of note this child also received chemotherapy that consists of etoposide, dexamethasone and cyclosporine A, as part of HLH-2004 protocol. ${ }^{5}$

EBV infection is the most common cause of infection-related HLH. It represents one-third of the infection related cases. ${ }^{6}$ Etoposide is the best available treatment option for EBV-related HLH and it has activity in histiocytic diseases and also inhibits EBV nuclear antigen.

Less commonly Leishmania donovani and Leishmania infantum have been reported to cause HLH. ${ }^{7}$ Liposomal amphotericin B, which is selectively undertaken by the reticuloendothelial cells, is the best available treatment for visceral leishmaniasis. ${ }^{8}$ However, visceral leishmaniasis and HLH are very similar in clinical presentations, which may lead to misdiagnosis and wrong therapy. ${ }^{9}$ Therefore, a careful investigation for the underlying cause of HLH should be performed. This case report raises the question when evidence of $\mathrm{EBV}$ infection is found, it should be interpreted carefully in HLH patients and thorough search for additional etiological agents should be carried out. Treatment of actual etiological agent can lead to complete cure while using current recommend chemotherapy and immunotherapy in patient with hidden infection may have deleterious effects. Clinicians should be aware about clinical entity of EBV and Leishmania co-infection in patients with HLH.

\section{Conclusions}

Even in low incidence countries for visceral leishmaniasis, the Leishmania infantum infection should be considered in any patient with HLH. In cases of EBV-related HLH a thoroughly search for additional agent triggering $\mathrm{HLH}$ should be performed.

\section{References}

1. Weitzman S. Approach to hemophagocytic syndromes. Hematology 2011;2011:178-83.

2. Rosado FGN, Kim AS. Hemophagocytic lymphohistiocytosis: an update on diagnosis and pathogenesis. Am J Clin Pathol 2013;139:713-27.

3. Henter JI, Horne A, Arico M, et al. HLH2004: diagnostic and therapeutic guidelines for hemophagocytic lymphohistiocytosis. Pediatr Blood Cancer 2007;48:124-31.

4. Koliou MG, Soteriades ES, Ephros M, et al. Hemophagocytic lymphohistiocytosis associated with Epstein Barr virus and Leishmania donovani coinfection in a child from Cyprus. J Pediatr Hematol Oncol 2008; 30:704-7.

5. Dominguez-Pinilla N, Baro-Fernández M, González-Granado LI. Hemophagocytic lymphohistiocytosis secondary to Epstein Barr virus and Leishmania co-infection in a toddler. J Postgrad Med 2015;61:44-5.

6. Janka G, Imashuku S, Elinder G, et al. Infection- and malignancy-associated hemophagocytic syndromes. Secondary hemophagocytic lymphohistiocytosis. Hematol Oncol Clin North Am 1998;12:435-44.

7. Cascio A, Pernice LM, Barberi G, et al. Secondary hemophagocytic lymphohistiocytosis in zoonoses. A systematic review. Eur Rev Med Pharmacol Sci 2012;16:1324-37.

8. Bern C, Adler-Moore J, Berenguer J, et al. Liposomal amphotericin B for the treatment of visceral leishmaniasis. Clin Infect Dis 2006;43:917-24.

9. Gagnaire MH, Galambrun C, Stéphan JL. Hemophagocytic syndrome: A misleading complication of visceral leishmaniasis in children: a series of 12 cases. Pediatrics 2000;106:E58. 